\title{
Evaluación de la violencia y deportividad en el deporte: un análisis bibliométrico
}

\section{Evaluation of violence and sportsmanship in sport: bibliometric analysis}

\section{Avaliação da violência e desportivismo no desporto: uma análise bibliométrica}

\author{
A. Sáenz ${ }^{1 *}$, F. Gimeno ${ }^{2}$, H. Gutiérrez ${ }^{3}$, D. Lacambra², R. Arroyo del Bosque y C. Marcén ${ }^{3}$
}

${ }^{1}$ Universidad del Pais Vasco, ${ }^{2}$ Universidad de Zaragoza, ${ }^{3}$ Universidad San Jorge de Zaragoza

\begin{abstract}
Resumen: Este trabajo presenta un análisis bibliométrico de las publicaciones sobre los instrumentos de evaluación de la deportividad, la agresividad y la violencia en contextos deportivos profesionales y en edad escolar, durante el período 1996 a 2013. Los documentos fueron identificados en las bases de datos SportDiscus, PsycINFO, Psicodoc y Dialnet, así como en publicaciones e informes de federaciones e instituciones deportivas. Han sido 530 los documentos analizados, de los cuales 35 presentan instrumentos de evaluación. Los documentos obtenidos fueron clasificados en 5 categorías: método de evaluación, constructos evaluados, agentes sociales evaluados, período temporal y contexto deportivo. Los resultados muestran que la herramienta más utilizada ha sido el cuestionario (76.19\%). En segundo lugar, los instrumentos analizados permiten evaluar variables psicológicas relacionadas con la deportividad (14.28\%), con la no deportividad (28.57\%) o con ambas (57.14\%). En tercer lugar, tienden a centrarse en la evaluación del deportista $(71.43 \%)$. En cuarto lugar, la mayoría permiten ser utilizados en un único momento temporal (85.71\%). Finalmente, los instrumentos permiten ser utilizados tanto en ámbitos deportivos profesionales como en edad escolar (66.66\%) o exclusivamente en edad escolar (33.33\%).

Palabras clave: Análisis bibliométrico, evaluación, agresividad, violencia, deportividad, deporte en edad escolar.

Abstract: This study presents a bibliometric analysis of printed publications on evaluation tools in sportsmanship, aggresivity and violence in a professional sports context and at school age during 2013. Records from databases SportDiscus, PsycINFO, Psicodoc and Dialnet, as well as printed publications and reports from federations and sports institutions have been used. 530 documents have been analized, out of which 35 show evaluation tools. The obtained records were classified into 5 catagories: methods of assessment, constructs and social actors assessed, seasonal period and sports context. The results show that the most used tool was the survey $(76.19 \%)$.
\end{abstract}

Secondly, the tools analized allowed the evaluation of psychological variables related to sportsmanship (14.28\%), anti-sportsmanship (28.57\%) and both $(57.14 \%)$. Thirdly, nearly all the tools are centred on the assessment of the sportsman (71.43\%). Fourthly, most of them are able to be used at any one single moment (85.71\%). Finally, many of the tools allow evaluation in both the professional and school setting $(66.66 \%)$ or entirely in the school setting (33.33\%).

Key words: Bibliometric analysis, evaluation, aggressiveness, violence, sportsmanship, sport at school age.

Resumo: Este trabalho apresenta uma análise bibliométrica das publicaçôes sobre os instrumentos de avaliação do desportivismo, da agressividade e da violência em contextos desportivos profissionais e em idade escolar, durante o período de 1996 a 2013. Os documentos foram identificados nas bases de dados SportDiscus, PsycINFO, Psicodoc e Dialnet, assim como em publicaçôes e relatórios de federaçôes e de instituiçôes desportivas. Foram analisados 530 documentos, dos quais 35 apresentam instrumentos de avaliaçáo. Os documentos obtidos foram classificados em 5 categorias: método de avaliação, modelos avaliados, agentes sociais avaliados, período temporal e contexto desportivo. Os resultados mostram que a ferramenta mais utilizada foi o questionário (76.19\%). Em segundo lugar, os instrumentos analisados permitem avaliar variáveis psicológicas relacionadas com o desportivismo (14.28\%), com o não desportivismo (28.57\%) ou com ambos (57.4\%). Em terceiro lugar, tendem a centrar-se na avaliaçáo do desportista $(71.43 \%)$. Em quarto lugar, a maioria permite ser utilizada num único momento temporal (85.71\%). Por fim, os instrumentos permitem ser utilizados tanto em âmbitos desportivos profissionais como em idade escolar (66.66\%) ou exclusivamente em idade escolar (33.33\%).

Palavras-chave: Análise bibliométrica, avaliação, agressividade, violência, desportivismo, desporto em idade escolar.

\section{Introducción}

El deporte se ha convertido en un hecho representativo de la sociedad actual. Según Bredemeier y Shields (1984), el deporte puede facilitar el desarrollo de cualidades personales y sociales. De la misma manera, también señalan que puede ser favorecedor de comportamientos no deportivos. En esta línea, estos autores indican que el deporte es un área de la interacción humana en el que la agresión algunas veces es rechazada, algunas veces condenada, pero muchas otras veces legitimada

\footnotetext{
Dirección para correspondencia [Correspodence address]: Alfredo Sáenz Ibáńez, Calle San Millán no 3 1º A, CP: 01006, Vitoria-Gasteiz (España). E-mail: saenzalfredo@hotmail.com
}

y tolerada. La literatura muestra cómo han sido diversos los instrumentos los que han pretendido conocer si la práctica deportiva se caracteriza por la presencia de comportamientos deportivos, por la presencia de comportamientos agresivos o violentos o por la presencia de ambos tipos de comportamientos de manera simultánea. Por ello, se ha realizado un análisis utilizando los indicadores bibliométricos con el fin de describir y analizar de forma estructurada los documentos hallados.

Este análisis responde a la necesidad de conocer las características específicas de los instrumentos utilizados para evaluar los constructos deportividad, agresividad y violencia 
en los contextos deportivos, haciendo especial hincapié en los utilizados en ámbitos deportivos educativos, concretamente en edad escolar. Una circunstancia que se observa en los estudios que han evaluado los constructos objeto de estudio es, según Rato, Ley y Durán (2009), que las evaluaciones que se realizan en los contextos deportivos, en ocasiones, carecen de contraste empírico alguno. Este hecho, como señala Turnbull (2002), impide un conocimiento orientado a una intervención preventiva posible y realista e imposibilita una toma de decisiones racional y empírica.

Concretamente, en los contextos deportivos en edad escolar, es donde se prioriza la construcción de la escala de valores de las personas y su desarrollo moral. Weinberg y Gould (2011), señalan que las experiencias que tienen los deportistas durante sus etapas formativas, pueden tener consecuencias transcendentales en su personalidad y desarrollo psicológico. Sin embargo, en los contextos deportivos profesionales y en el deporte en edad escolar en particular, el fenómeno de la deportividad o la falta de ésta es un tema relevante en la sociedad actual. Los constructos deportividad, agresividad y violencia, están vinculados a diferentes variables. Por un lado, a los agentes que rodean a los jóvenes deportistas (Weinberg y Gould, 2010). Diferentes autores señalan la importancia de las influencias sociales; los iguales (Ullrich-French y Smith, 2009), los profesores de educación física (Berengüí y Garcés de los Fayos, 2007), los entrenadores (Conroy y Coatsworth, 2006; Horn, 2008), los padres/espectadores (Leo et al., 2009; Teques y Serpa, 2009; Wuerth, Lee y Alfermann, 2004), los árbitros (Dosil, 2003; González-Oya y Dosil, 2007; Ramírez, Alonso-Arbiol, Falcó y López, 2006), los medios de comunicación (Simon, 1991) y los representantes institucionales (Tenenbaum et al., 1997, citado en Weinberg y Gould, 2010), a la hora de explicar los comportamientos prosociales y antisociales en contextos deportivos. Sin embargo, como señala Greedorfer (1992), la mayoría de investigaciones que han pretendido explicar los comportamientos deportivos y no deportivos, han ignorado la importancia de las influencias sociales señaladas, especialmente en los contextos deportivos en edad escolar.

Una segunda variable a tener en cuenta, a la hora de explicar los constructos objeto de estudio, son las situaciones antecedentes; el contexto o la situación de un partido, el marcador o la posición parcial durante la competición (Gimeno, 2011). En tercer lugar, se deben tener en cuenta variables psicológicas: el estado emocional del deportista (Roberts, 1995; Tenenbaum, Fogarty y Jackson, 1999), sus orientaciones motivacionales (Harwood, Cumming y Fletcher, 2004; Vallerand y Bissonnette, 1992), el autoconcepto (Moreno, 2008; Slutzky y Simpkins, 2009; Stewart y Corbin, 1989), entre otras.

En esta perspectiva multivariante de la deportividad, la violencia y la agresividad en el deporte, son especialmente relevantes los marcos teóricos que a continuación se presentan, y en los que se han apoyado las investigaciones para explicar este fenómeno en el ámbito del deporte ; las teorías explicativas del aprendizaje (Weinberg y Gould, 2010; Zinsser, Bunker y Willians, 2001); la teoría de la agresión-frustración (Volkamer, 1971); la teoría estructural del desarrollo moral (Bredemeier y Shields, 1984; Romand, Pantaléon y Cabagno, 2009; Weiss y Bredemeier, 1991); la teoría motivacional de la orientación de metas de logro (Kavussanu y Roberts, 2001; Miller, Roberts y Ommundsen, 2005; Nicholls, 1989; Stephens y Bredemeier, 1996) y la teoría de la autodeterminación (Vallerand, 1987). Sin embargo, como señala Roberts (1995), ningún marco teórico ofrece una explicación completa de las causas de las conductas deportivas o no deportivas.

Así, se concluye que la deportividad, la agresividad y la violencia tienen una naturaleza multicausal o multivariante (Gimeno et al., 2011), a la cual, a partir de una evaluación rigurosa de las variables que influyen en los contextos deportivos, hay que dar respuesta. En este sentido, al igual que otras variables psicológicas que influyen en la práctica deportiva, los constructos anteriormente señalados, deben poder evaluarse correctamente con objeto de controlar y, si es posible, incrementar o reducir su gradación.

\section{Influencia de variables psicosociales y contextuales en la deportividad, la agresividad y la violencia}

Son diversas las variables que hay que tener en cuenta a la hora de explicar los constructos deportividad, agresividad y violencia. Según Martens (1982), no existen definiciones universalmente aceptadas relacionadas con la deportividad, sino que habría que identificar de manera específica las conductas deportivas, su conexión con cada deporte, con el nivel de juego de los deportistas y la edad de éstos. En la misma línea, se han propuesto diferentes explicaciones a la hora de abordar las conductas violentas y agresivas en los contextos deportivos: la intencionalidad (Gill, 2000), el tipo de refuerzo primario que obtiene el deportista al realizar la conducta violenta (Tenenbaum, Stewart, Singer y Duda, 1997); el conjunto de pensamientos, actitudes y comportamientos del deportista (Pelegrín y Garcés de Los Fayos, 2007, p. 17). Todas estas variables a tener en cuenta en el estudio de la deportividad, la agresividad y la violencia, coinciden en que los comportamientos no deportivos hacen referencia a reacciones desajustadas como por ejemplo, insultar, amenazar, desafiar, criticar, pegar, empujar o escupir, entre otras acciones. Según Goldstein (1999), cada uno de estos actos violentos puede deberse a múltiples causas. Atendiendo a los motivos de las conductas antisociales, Garmezy (1983) y Rutter (1990), señalan que se producen debido a una serie de factores de riesgo. Estos factores son denominados variables predictoras, ya que predicen las distintas causas que parecen generar las conductas violentas. 


\section{Evaluación de modalidades deportivas}

El deporte se ha convertido en un hecho representativo de la sociedad actual. El deporte afecta de manera diferente a un gran número de personas, influyendo en su estilo de vida, en sus relaciones interpersonales, en la adquisición de valores, etc. Diversos autores (Blázquez, 1999; Cagigal, 1981; Matveev, 1980; García Ferrando, 2001; Robles, Abad y Giménez, 2009; Vargas 2007), han planteado clasificaciones para diferenciar las modalidades deportivas, según la función que los practicantes otorgan al deporte. Como señala Cagigal (1983), el deporte es un fenómeno cultural permeable a las transformaciones del entorno y por tanto, hay que considerarlo desde una perspectiva polisémica (Brohm, 1976), "como un sistema complejo con diversidades y contradicciones" (Puig y Heinemann, 1991, p. 125). Según Willimczik (2009), continuamente surgen enfoques de modalidades deportivas nuevas, que aunque no se ajustan a concepciones clásicas de deporte, se hace necesario tenerlas en cuenta. Sin embargo, el deporte se muestra como una realidad cada vez más difícil de acotar. Por ello, en la presente investigación, a partir de un estudio piloto de los documentos hallados en el análisis bibliométrico, se parte de una definición de deporte como sistema abierto (Dietrich y Heinemann, 1989; Heinemann, 1991).

El deporte profesional tiene la finalidad de conseguir la victoria. Éste produce espectáculo, gusta al público y es rentable. Entre las características del deporte profesional destaca que el deportista busca la mejor marca posible, un récord, la gloria pública, prestigio, enriquecimiento. Desde esta perspectiva, el deporte interviene en la vida social, política y económica de los individuos. En este ámbito, el deporte se convierte en cultura de masas, en un medio para producir excitación en la sociedad, en un negocio, en un espectáculo en el que el resultado de la competición es trascendental. A través del deporte profesional, se pueden transmitir a otros ámbitos, como al deporte educativo, contravalores: ganar a toda costa, humillación, venganza, etc. Pero también posee un gran potencial formativo, que canalizado de forma correcta, puede servir para educar y formar ciudadanos más cívicos y mejor educados. Como señala Durán (1996), los responsables de los espectáculos deportivos (entrenadores, directivos de clubes, representantes institucionales, etc.) tienen una enorme responsabilidad como líderes sociales, hacia la sociedad en general y hacia los jóvenes deportistas en particular.

Por otro lado, el deporte educativo, que abarca el período en edad escolar del joven deportista, se entiende como proceso de socialización. Este término se ha definido como "el proceso por el cual los individuos aprenden destrezas, normas y valores para el desempeño de roles sociales presentes o anticipados" (McPherson y Brown, 1988, p. 267). El Consejo Superior de Deportes Espańol denomina deporte en edad escolar a todas aquellas actividades que, de forma organizada se celebran fuera del programa de la asignatura de Educación Física en los centros escolares, clubes, asociaciones, etc. y, en las que participan deportistas en edad escolar. Según Bredemeier y Shields (1984), el deporte educativo constituye un contexto ideal donde se facilitan oportunidades únicas para desarrollar cualidades personales y sociales. En la sociedad actual existe de manera general una creciente preocupación por la adquisición de valores entre los jóvenes deportistas. Por ello, desde los diferentes contextos deportivos educativos (clubes deportivos, sociedades anónimas deportivas, centros escolares, etc.), se debe orientar a los jóvenes hacia un deporte formativo, orientado además de a la adquisición de las destrezas propias del deporte, a la adquisición de valores. Estos son "objetivos transituacionales deseables, que varían en importancia y que sirven como principios de guía en las vidas de las personas" (Schwartz, 1996, p. 2).

Considerando la diferenciación precedente entre las modalidades de deporte profesional y educativo, en la presente investigación se diferencia la modalidad profesional y la modalidad educativa en edad escolar en los contextos deportivos.

A lo largo de la historia de la psicología del deporte, se han propuesto un abanico de instrumentos que han pretendido evaluar conceptos relacionados con los constructos deportividad, agresividad y violencia. A continuación, con el propósito de conocer las características de los instrumentos que han evaluado los constructos seńalados, se presenta la búsqueda que se ha realizado para la compilación de los documentos que recogen los instrumentos de evaluación utilizados en contextos deportivos profesionales y/o en edad escolar.

\section{Método}

En el presente estudio, se ha llevado a cabo un análisis bibliométrico (Spinak, 1996), para estudiar las características del uso y creación de documentos, relacionados con la evaluación de los constructos deportividad, agresividad y violencia. El 20 de diciembre de 2013 se consultaron cuatro bases de datos internacionales; Sportdiscus, PsycINFO, Psicodoc y Dialnet. Las dos primeras, principalmente recogen un mayor número de referencias de lengua inglesa y las dos últimas, de lengua castellana. Los descriptores utilizados en la búsqueda han sido en Sportdiscus y PsycINFO: evaluation* ${ }^{*}$ and violence* ${ }^{*}$ or sportsmanship ${ }^{*}$ and sport* En Dialnet y Psicodoc: evaluación* y violencia* o deportividad* y deporte*. Atendiendo a la casuística de cada base de datos se ha acotado la búsqueda en PsycINFO, desde el año 1996 hasta el 2013, y en Sport Discus de diciembre de 1996 a diciembre de 2013. De la misma manera, tanto Dialnet como Psicodoc no permiten delimitar el período temporal, por lo que a partir de los descriptores seńalados, se han recogido los documentos encontrados en cada una de ellas. La búsqueda en las bases de datos se ha realizado en la opción "title" ó "título", según la base de datos. El moti- 
vo es porque utilizando otros campos, aumenta sobremanera el número de documentos o se reduce significativamente, dejando de aparecer documentos interesantes para la revisión.

En una primera fase, dos observadores llevaron a cabo el visionado de los documentos, a partir del cual se establecieron las categorías: idioma en el que están escritos, media de autores, procedencia en función del país, eje temático, tipología, método de evaluación, agentes evaluados, momento de la acción evaluadora, ámbito y constructos evaluados. En una segunda fase, cada observador realizó de forma individual la categorización de los documentos. El análisis se llevó a cabo con el programa $\mathrm{N}$-vivo versión 9. Este es un programa diseñado para el análisis de datos cualitativos que facilita la codificación y análisis de los documentos obtenidos de las bases de datos. Permite almacenar, organizar y obtener informes resumidos de los datos más significativos que emergen del análisis. Además, permite combinar una doble dimensión en el análisis integrando una perspectiva narrativa (más cualitativa) y analítica (más cuantitativa). Finalmente, en una tercera fase, siguiendo la fórmula de porcentaje de acuerdos utilizada por Bellack, Klicbard, Hyman y Smith (1966), se contrastaron los datos obtenidos por cada observador para determinar la fiabilidad intraobservador. Los resultados muestran que el índice de concordancia fue del $88 \%$.

Además, se ha revisado documentación vinculada a estamentos nacionales; el Consejo Superior de Deportes de España (CSD) y la Asociación de Federaciones Deportivas Españolas (AFEDES). De la misma manera, se ha revisado información aportada desde estamentos internacionales; la "General Association of International Sports Federations" (GAISF) y el Comité Olímpico Internacional (COI).

A partir de los documentos que recogen las bases de datos y la literatura no científica publicada en estamentos nacionales e internacionales, a continuación se muestran los resultados de la búsqueda relacionados con el idioma, el número de autores por documento y la procedencia de éste. Posteriormente, se presenta información sobre contenidos relacionados específicamente con instrumentos que evalúan la deportividad, la agresividad y la violencia en el deporte en general, y de manera específica en contextos deportivos en edad escolar.

Finalmente, a la hora de considerar los parámetros del estudio bibliométrico, se han tenido en cuenta las directrices de la declaración PRISMA (Liberati, et al., 2009; Urrútia y Bon fill, 2010). De esta manera, se ha pretendido ser sistemáticos y explícitos en la revisión.

\section{Resultados}

Los documentos hallados en las bases de datos revisadas fueron 535 (249 en Sportdiscus, 241 en Psyquinfo, 40 en Psicodoc y 5 en Dialnet). Entre los 535 documentos encontrados, se han hallado 5 duplicados exactos. Por tanto, han sido 530 los documentos analizados.

Por otro lado, se ha revisado documentación vinculada a estamentos deportivos nacionales e internacionales. Estos estamentos, mayoritariamente, recogen la legislación que existe relacionada con las leyes vigentes contra la violencia en el deporte. Esporádicamente, presentan acciones de prevención secundaria, es decir, actuaciones una vez que han ocurrido los incidentes no deportivos. Sin embargo, no se ha encontrado información relacionada con instrumentos que evalúen los constructos objeto de estudio.

En la Tabla 1 se muestra la frecuencia y el porcentaje obtenido en relación a: el idioma en el que está escrito el documento, su lugar de publicación, el promedio de autores y el año de publicación.

Tabla 1. Frecuencia y porcentaje de los documentos en función del idioma, la procedencia del documento, el promedio de autores y el año de publicación

\begin{tabular}{cccccccccccc}
\hline Idioma & $\mathbf{n}$ & $\%$ & Procedencia & $\mathbf{n}$ & $\%$ & $\mathbf{N}^{\circ}$ autores & $\mathbf{n}$ & $\%$ & Año publicación & $\mathbf{N}$ & $\%$ \\
\hline Inglés & 468 & 88.2 & EEUU & 246 & 46.4 & 1 & 208 & 38.9 & 2009 & 48 & 9.1 \\
Castellano & 49 & 9.3 & $*$ & 140 & 26.4 & 2 & 101 & 18.9 & 2011 & 42 & 7.9 \\
Francés & 8 & 1.5 & España & 44 & 8.3 & $*$ & 62 & 11.6 & 2003 & 41 & 7.7 \\
Alemán & 3 & 0.6 & Reino Unido & 32 & 6 & 3 & 58 & 10.8 & 2005 & 39 & 7.4 \\
Turco & 1 & 0.2 & Netherland & 19 & 3.6 & 4 & 50 & 9.3 & 2010 & 38 & 7.2 \\
Portugués & 1 & 0.2 & Alemania & 19 & 3.6 & 5 & 21 & 3.9 & 2008 & 35 & 6.7 \\
& & & Francia & 7 & 1.3 & 6 & 20 & 3.7 & 2001 & 34 & 6.4 \\
& & & Canadá & 5 & 0.9 & 7 & 7 & 1.3 & 2006 & 33 & 6.2 \\
& & & Suiza & 3 & 0.6 & 8 & 3 & 0.6 & 2007 & 33 & 6.2 \\
& & & Australia & 3 & 0.6 & 9 & 3 & 0.6 & 2013 & 28 & 5.3 \\
& & & Italia & 2 & 0.4 & 10 & 2 & 0.4 & 2004 & 25 & 4.7 \\
& & & Colombia & 2 & 0.4 & & & & 1999 & 22 & 4.1
\end{tabular}




\begin{tabular}{|c|c|c|c|c|c|c|c|c|c|c|c|}
\hline Idioma & $\mathbf{n}$ & $\%$ & Procedencia & $\mathbf{n}$ & $\%$ & No autores & $\mathbf{n}$ & $\%$ & Año publicación & $\mathrm{N}$ & $\%$ \\
\hline & & & Chile & 2 & 0.4 & & & & 2012 & 22 & 4.1 \\
\hline & & & Brasil & 2 & 0.4 & & & & 1998 & 21 & 4 \\
\hline & & & Israel & 1 & 0.2 & & & & 2002 & 21 & 4 \\
\hline & & & Turquía & 1 & 0.2 & & & & 1996 & 18 & 3.4 \\
\hline & & & Noruega & 1 & 0.2 & & & & 1997 & 15 & 2.8 \\
\hline & & & Grecia & 1 & 0.2 & & & & 2000 & 15 & 2.8 \\
\hline Total & 530 & 100 & & 530 & 100 & & 530 & 100 & & 530 & 100 \\
\hline
\end{tabular}

(*) Sin especificar en la base de datos

Como se puede observar en la tabla anterior, el 88.2\% de los documentos de las bases de datos revisadas, están escritos en lengua inglesa. En menor medida, se realizan publicaciones en otras lenguas; castellano, 9.3\% o francés, 1.5\%. En segundo lugar, un $46.4 \%$ son publicados en los EEUU. El porcentaje de publicaciones procedentes de Espańa, es del 8.3\%. Estos datos se deben a que, como se ha seńalado anteriormente, las bases de datos Psicodoc y Dialnet, son de origen latino. En tercer lugar, un 38.9\% de los documentos son publicados por un único autor. Finalmente, en el periodo temporal analizado, desde el año 1996 al ańo 2013, el 9.1\% de los documentos fueron publicados en el año 2009.

En la Tabla 2 se muestra la frecuencia y el porcentaje obtenido en relación: al tipo de documento y las temáticas que aparecen en las bases de datos revisadas, a partir de los descriptores anteriormente señalados.

Tabla 2. Frecuencia y porcentaje de los documentos según su tipología y eje temático.

\begin{tabular}{|c|c|c|c|c|c|}
\hline Tipo de documento & $\mathrm{n}$ & $\%$ & Eje temático & $\mathrm{n}$ & $\%$ \\
\hline Artículo & 422 & 79.6 & Género & 124 & 23.4 \\
\hline Libro & 53 & 10 & Otros & 80 & 15.1 \\
\hline Tesis doctoral & 43 & 8.1 & Ámbitos educativos & 48 & 9.1 \\
\hline Referencia genérica & 6 & 1.1 & Premios deportividad & 44 & 8.3 \\
\hline Capítulo libro & 4 & 0.7 & Programas deportividad & 41 & 7.7 \\
\hline Vídeo/DVD & 1 & 0.2 & Evaluación deporte & 35 & 6.7 \\
\hline \multirow[t]{11}{*}{ Acta conferencia } & 1 & 0.2 & Deportividad & 33 & 6.2 \\
\hline & & & Juvenil & 31 & 5.9 \\
\hline & & & Teoría violencia & 23 & 4.3 \\
\hline & & & Medios comunicación & 12 & 2.3 \\
\hline & & & Nińos & 10 & 1.9 \\
\hline & & & Drogodependencias & 10 & 1.9 \\
\hline & & & Leyes & 9 & 1.7 \\
\hline & & & Documentos de opinión & 8 & 1.5 \\
\hline & & & Delincuencia & 8 & 1.5 \\
\hline & & & Laboral & 7 & 1.3 \\
\hline & & & Deporte adaptado & 7 & 1.3 \\
\hline Total & 530 & 100 & & 530 & 100 \\
\hline
\end{tabular}

Tal y como se refleja en la Tabla 2., la mayor parte de los documentos encontrados son artículos, el 79.6\%. En menor medida los documentos se refieren a libros o tesis doctorales, el $10 \%$ y $8.1 \%$ respectivamente. Finalmente, como se puede observar, la mayor parte de los documentos abordan la temática de género, el 23.4\%. De la misma manera, se puede observar que únicamente 35 de los documentos, el 6.7\%, están relacionados con la evaluación de contextos deportivos.

A continuación (ver Tabla 3), se especifican los instrumentos de evaluación identificados en los 35 documentos cuyo eje temático es "evaluación deporte". Concretamente, se presenta la denominación de los instrumentos, se especifica el método de evaluación utilizado, se concretan los constructos evalua- 
dos, los agentes que se evalúan, el período temporal durante el que se realiza la evaluación y el ámbito en el que el instru- mento permite ser utilizado, profesional, en edad escolar o en ambos.

Tabla 3. Características de los instrumentos de evaluación utilizados para la evaluación de la deportividad y/o no deportividad en el deporte profesional y en edad escolar.

\begin{tabular}{|c|c|c|c|c|c|c|}
\hline Instrumento & $\begin{array}{c}\text { Método } \\
\text { evaluación }\end{array}$ & $\begin{array}{l}\text { Constructo } \\
\text { evaluado }(*)\end{array}$ & $\begin{array}{c}\text { Agente } \\
\text { evaluado }\end{array}$ & $\begin{array}{l}\text { Temporalización } \\
\text { de la evaluación }\end{array}$ & $\begin{array}{c}\text { Ámbito } \\
(* *)\end{array}$ & $\begin{array}{l}\text { Características } \\
\text { psicométricas }\end{array}$ \\
\hline $\begin{array}{l}\text { Actas arbitrales (González-Oya y } \\
\text { Dosil, 2004; Lefebre y Passer, 1974) }\end{array}$ & Informe & ND & $\begin{array}{l}\text { deportistas y } \\
\text { entrenadores }\end{array}$ & longitudinal & DE y DP & $\begin{array}{l}\text { Sin características } \\
\text { psicométricas }\end{array}$ \\
\hline $\begin{array}{l}\text { Prosocial and Antisocial Behavior in } \\
\text { Sport Scale (Kavussanu y Boardley, } \\
\text { 2009) }\end{array}$ & Cuestionario & D y ND & deportistas & puntual & DE y DP & $\begin{array}{l}\text { Factor } 1 \mathrm{a}=.86 \\
\text { Factor } 2 \mathrm{a}=.83 \\
\text { Factor } 3 \mathrm{a}=.74 \\
\text { Factor } 4 \mathrm{a}=.86\end{array}$ \\
\hline $\begin{array}{l}\text { Registro de Evaluación de Partidos } \\
\text { de Fútbol (Gimeno et al., 2007) }\end{array}$ & Cuestionario & D y ND & $\begin{array}{l}\text { deportistas } \\
\text { entrenadores } \\
\text { espectadores } \\
\text { árbitro }\end{array}$ & longitudinal & $\mathrm{DE}$ & $\begin{array}{l}\text { Escala } 1 \mathrm{a}=.87 \\
\text { Escala } 2 \mathrm{a}=.80 \\
\text { Escala } 3 \mathrm{a}=.76\end{array}$ \\
\hline $\begin{array}{l}\text { Cuestionario de Intenciones y Com- } \\
\text { portamientos Antisociales en Fútbol } \\
\text { (García Calvo, 2006) }\end{array}$ & Cuestionario & ND & deportistas & puntual & DE y DP & $\begin{array}{l}\text { Factor } 1 \mathrm{a}=.84 \\
\text { Factor } 2 \mathrm{a}=.82 \\
\text { Factor } 3 \mathrm{a}=.86\end{array}$ \\
\hline $\begin{array}{l}\text { Cuestionario de Actitudes Antide- } \\
\text { portivas y Violentas para Deportistas } \\
\text { (Pelegrín, 2005) }\end{array}$ & Cuestionario & ND & deportistas & puntual & DE y DP & $\begin{array}{c}\text { Cuestionario } \\
\mathrm{a}=.84\end{array}$ \\
\hline $\begin{array}{l}\text { Escala de Actitudes Competitivas } \\
\text { (Lakie, 1964) }\end{array}$ & Cuestionario & $\begin{array}{c}\text { D y ND } \\
\text { Listado "ganar } \\
\text { a toda costa" }\end{array}$ & deportistas & puntual & DE y DP & $\begin{array}{l}\text { Validez por grupo } \\
\text { de expertos }\end{array}$ \\
\hline $\begin{array}{l}\text { Multidimensional Sportpersonship } \\
\text { Orientations (Vallerand, 1997) }\end{array}$ & Cuestionario & $\mathrm{D}$ & deportistas & puntual & DE y DP & $\begin{array}{c}\text { Cuestionario } \\
\mathrm{a}=.72\end{array}$ \\
\hline $\begin{array}{l}\text { Sports Values Questionnaire (Lee, } \\
\text { 1996) }\end{array}$ & Cuestionario & $\begin{array}{l}\text { D } \\
\text { Listado de } \\
\text { valores }\end{array}$ & deportistas & puntual & $\mathrm{DE}$ & $\begin{array}{l}\text { Cuestionario } \\
\mathrm{a}=.83\end{array}$ \\
\hline $\begin{array}{l}\text { Horroks Prosocial Play Behavior Inven- } \\
\text { tory (Horroks, 1979) }\end{array}$ & Cuestionario & D y ND & deportistas & puntual & $\mathrm{DE}$ & $\begin{array}{l}\text { Sin características } \\
\text { psicométricas }\end{array}$ \\
\hline
\end{tabular}

* Deportividad (D) No Deportividad (ND)

** Deporte Escolar (DE) Deporte Profesional (DP)

Tabla 3. Características de los instrumentos de evaluación utilizados para la evaluación de la deportividad y/o no deportividad en el deporte profesional y en edad escolar (continuación)

\begin{tabular}{|c|c|c|c|c|c|c|}
\hline Instrumento & $\begin{array}{c}\text { Método } \\
\text { evaluación }\end{array}$ & $\begin{array}{l}\text { Constructo } \\
\text { evaluado }(*)\end{array}$ & $\begin{array}{l}\text { Agente } \\
\text { evaluado }\end{array}$ & $\begin{array}{l}\text { Temporalización } \\
\text { de la evaluación }\end{array}$ & $\begin{array}{c}\text { Ámbito } \\
(* *)\end{array}$ & $\begin{array}{l}\text { Características } \\
\text { psicométricas }\end{array}$ \\
\hline $\begin{array}{l}\text { Cuestionario de disposición al enga- } \\
\text { ño deportivo (Ponseti et al., 2012) }\end{array}$ & Cuestionario & ND & deportistas & puntual & DE y DP & $\begin{array}{c}\text { Cuestionario } \\
\mathrm{a}=.78\end{array}$ \\
\hline $\begin{array}{l}\text { Escala de Actitudes de Juego Limpio } \\
\text { (Cruz, 1996) }\end{array}$ & Cuestionario & D y ND & deportistas & puntual & DE y DP & $\begin{array}{l}\text { Escala } 1 \mathrm{a}=.74 \\
\text { Escala } 2 \mathrm{a}=.66 \\
\text { Escala } 3 \mathrm{a}=.60\end{array}$ \\
\hline $\begin{array}{l}\text { Cuestionario de situaciones hipo- } \\
\text { téticas de deportividad (Vallerand, } \\
\text { Deshaies y Cuerrier, 1997) }\end{array}$ & Cuestionario & $\mathrm{D}$ & deportistas & puntual & DE y DP & $\begin{array}{c}\text { ANOVA } \\
\text { Relación entre in- } \\
\text { tención de compor- } \\
\text { tamiento y preocupa- } \\
\text { ción hacia el oponente }\end{array}$ \\
\hline $\begin{array}{l}\text { Sportmanship Coaching Behaviors } \\
\text { Scale (Bolter y Weiss, 2012) }\end{array}$ & Cuestionario & D y ND & entrenadores & puntual & $\mathrm{DE}$ & $\begin{array}{l}8 \text { sub-escalas } \\
\mathrm{a}=.78-.95\end{array}$ \\
\hline
\end{tabular}




\begin{tabular}{|c|c|c|c|c|c|c|}
\hline Instrumento & $\begin{array}{c}\text { Método } \\
\text { evaluación }\end{array}$ & $\begin{array}{l}\text { Constructo } \\
\text { evaluado }\left(^{*}\right)\end{array}$ & $\begin{array}{c}\text { Agente } \\
\text { evaluado }\end{array}$ & $\begin{array}{l}\text { Temporalización } \\
\text { de la evaluación }\end{array}$ & $\begin{array}{c}\text { Ámbito } \\
(* *)\end{array}$ & $\begin{array}{l}\text { Características } \\
\text { psicométricas }\end{array}$ \\
\hline $\begin{array}{l}\text { McMahan Sportsmanship Attitude } \\
\text { Scale (McMahan, 1978) }\end{array}$ & Cuestionario & D y ND & entrenadores & puntual & $\mathrm{DE}$ & $\begin{array}{l}\text { ANOVA Relación } \\
\text { entre el género, cla- } \\
\text { sificación en la liga y } \\
\text { ańos de experiencia }\end{array}$ \\
\hline $\begin{array}{l}\text { Codes of Conduct for Coaches (Green- } \\
\text { well et al., 2001) }\end{array}$ & Cuestionario & $\mathrm{D}$ & entrenadores & puntual & $\mathrm{DE}$ & $\begin{array}{c}\text { Sub-escala deporti- } \\
\text { vidad } \\
\mathrm{a}=.86\end{array}$ \\
\hline $\begin{array}{l}\text { Measure of Moral Judgment, Reason } \\
\text { and Intention (Gibbons et al., 1995) }\end{array}$ & $\begin{array}{l}\text { Cuestionario, } \\
\text { registro } \\
\text { observación } \\
\text { y entrevista }\end{array}$ & D y ND & deportistas & puntual & $\mathrm{DE}$ & $\begin{array}{c}\text { Cuestionario } \\
\mathrm{a}>.80\end{array}$ \\
\hline $\begin{array}{l}\text { Instrumento de Observación de } \\
\text { Fairplay en Fútbol (Borrás, et al., } \\
\text { 2003; Cruz et al., 1996) }\end{array}$ & $\begin{array}{c}\text { Registro } \\
\text { observación }\end{array}$ & $\begin{array}{l}\text { D y ND } \\
\text { Grabación } \\
\text { de partidos } \\
\text { de fútbol }\end{array}$ & $\begin{array}{l}\text { deportistas } \\
\text { árbitro }\end{array}$ & longitudinal & 1 y 2 & $\begin{array}{l}\text { Kappa de Cohen } \\
\quad=.79\end{array}$ \\
\hline \multicolumn{7}{|l|}{${ }^{*}$ Deportividad (D) No Deportividad (ND) } \\
\hline ional & & & & & & \\
\hline
\end{tabular}

Atendiendo al ámbito profesional y en edad escolar de forma conjunta, se observa en la tabla anterior que la herramienta más utilizada por los investigadores para la evaluación de la incidencia de actitudes, valores y comportamientos deportivos, violentos y agresivos, ha sido el cuestionario. En menor medida se ha utilizado el registro de observación, la entrevista o el informe. En segundo lugar, estos instrumentos permiten evaluar variables psicológicas relacionadas con la deportividad; la "Multidimensional Sportpersonship Orientations (MSOS)” (Vallerand, 1997), versión traducida al castellano por Martín-Albo, J., Núñez, J., Navarro, J. y González, V. (2006), el Cuestionario de situaciones hipotéticas de deportividad (Vallerand, Deshaies y Cuerrier, 1997) o The Sports Values Questionnaire (SVQ) (Lee, 1996). Con la no deportividad; el Cuestionario de Intenciones y Comportamientos Antisociales en Fútbol (García Calvo, 2006), el Cuestionario de Actitudes Antideportivas y Violentas para Deportistas (Pelegrín, 2005), el Cuestionario de disposición al engaño deportivo (Ponseti et al., 2012). O con ambas; la Escala de Actitudes de Juego Limpio (Cruz, 1996), el "Prosocial and Antisocial Behavior in Sport Scale (PABSS)" (Kavussanu y Boardley, 2009), la "Competitive Attitude Scale (CAS)" (Lakie, 1964). La mayoría de los instrumentos que se presentan permiten ser utilizados tanto en ámbitos deportivos profesionales, como en edad escolar. En tercer lugar, se puede observar cómo mayoritariamente los instrumentos se centran exclusivamente en realizar la evaluación de los deportistas. Son escasos los que permiten la evaluación de otros agentes; entrenadores, espectadores o árbitros. En cuarto lugar, la mayoría de los instrumentos permiten ser utilizados en un único momento temporal. En quinto lugar, son pocos los instrumentos los que de forma específica permiten la evaluación del deporte en edad escolar. Finalmente, se presentan las características psicométricas de los instrumentos.

Han sido diversos los instrumentos los que de manera específica se han utilizado para la evaluación de los contextos deportivos en edad escolar (Bolter y Weiss, 2012; Gibbons, Ebbeck y Weiss, 1995; Gimeno et al., 2007; Greenwell et al., 2001; Horroks, 1979; Lee, 1996; McMahan, 1978). Las características de estos instrumentos se presentan a continuación. El Registro de Evaluación de Partidos de Fútbol (REPF) (Gimeno et al., 2007; Sáenz, 2010), específico para el deporte del fútbol, consta de cuatro partes. La primera, permite la calificación del partido en su conjunto y la valoración simultánea del comportamiento de entrenadores, deportistas, árbitros y espectadores. En una segunda parte, a partir de un listado de conductas y actitudes relacionadas con la deportividad y con la no deportividad, el árbitro y los dos entrenadores señalan, una vez finalizado el partido, aquellas que han ocurrido en el terreno de juego y en la grada. Estas conductas “explican" las valoraciones de carácter global reflejadas en la primera parte del registro. En una tercera parte, el árbitro recoge las sanciones que ha mostrado. Y finalmente, en una cuarta parte se pueden señalar otras actitudes y conductas no recogidas en el cuestionario. Los ítems de las tres escalas del instrumento presentan una capacidad discriminativa adecuada y una alta consistencia interna: alfa de Cronbach $=.87$, .80 y .76, respectivamente. El segundo de los instrumentos, "Horrocks Prosocial Play Behavior Inventory (HPPBI)" (Horroks, 1979), permite investigar la relación de ciertos comportamientos prosociales de deportistas juveniles respecto a; su desarrollo moral, la participación deportiva juvenil y las percepciones que tienen sobre deportividad. El instrumento refleja 10 conductas deportivas y antideportivas que suelen desarrollar los jóvenes deportistas en los contextos deportivos 
(eg. discuto con otros, desobedezco las reglas, ayudo a los demás, entre otras). Estos comportamientos son evaluados por un observador en una escala de 4 puntos. Los datos obtenidos a partir de este instrumento no son todo lo esclarecedores que se pretendía, ya que no permite la justificación de los actos realizados por los deportistas. El tercer instrumento que se presenta, pretende medir el juicio moral, el razonamiento moral y la intención del joven deportista (Gibbons, Ebbeck y Weiss, 1995). El juicio corresponde a la decisión de si un acto es correcto o no. La razón moral explica las causas que la persona esgrime para llevar a cabo una determinada acción. Y por último, la intención moral hace referencia a la posibilidad de que el individuo vuelva a repetir un comportamiento en el futuro. Este instrumento se basa en los 10 comportamientos observados en la herramienta anterior. Y además, se le añadieron tres preguntas. Gibbons et al. (1995), demostraron que este instrumento tiene una buena consistencia interna, con un valor de Alpha de Cronbach superior a .80. Asimismo, esta herramienta ha sido utilizada en investigaciones posteriores (Dunn y Dunn, 1999), confirmando su fiabilidad.

Un cuarto instrumento, es "The Sports Values Questionnaire (SVQ)” (Lee, 1996). Con el SVQ se pretende estudiar la adquisición de valores y actitudes en los jóvenes deportistas. Este instrumento, inicialmente está formado por 23 ítems. Cada ítem corresponde a un valor excepto cuatro valores que están reflejados en dos ítems. Así pues mide 19 valores relevantes en el deporte: animar, autorrealización, mantenimiento de contrato, imagen pública, conformidad, compañerismo, imitar modelos, justicia, logro personal, obediencia, forma física, búsqueda de sensaciones, deportividad, mejora de habilidades, aceptación, diversión, cohesión de equipo, ganar y ayudar. Las opciones de respuesta a cada ítem se ofrecen mediante una escala de 7 puntos (-1 a 5). En su versión espańola el SVQ-E-R (Torregrosa, 2002), presenta un alfa de Cronbach de .83 que puede considerarse un valor satisfactorio

El "Sportmanship Coaching Behaviors Scale (SCBS)" (Bolter y Weiss, 2012), se centra en recoger las conductas de los entrenadores en edad escolar. Este instrumento consta de 40 ítems, divididos en ocho tipos de comportamiento relacionados con las conductas y actitudes de los entrenadores: (a) establece expectativas de deportividad, (b) refuerza la deportividad, (c) castiga la conducta antideportiva, (d) discute la deportividad, (e) enseña deportividad, (f) presenta modelos deportividad, (g) presenta modelos de conducta antideportiva, y (h) prioriza el ganar sobre la deportividad. Este instrumento presenta un alfa de Cronbach en las sub-escalas de .78 y .95. El "Codes of Conduct for Coaches" (Greenwell et al., 2001), pretende conocer cómo los entrenadores actúan de manera deportiva y promueven el espíritu deportivo. Consta de siete sub-escalas, siendo una de ellas la de deportividad. Esta escala consta de nueve ítems. El alfa de Cronbach de esta sub-escala es de .86 . En el resto de sub-escalas es entre .70 y .97.

Finalmente, la "McMahan Sportsmanship Attitude Scale" (McMahan, 1978). El instrumento evalúa el comportamiento de los entrenadores a partir del comportamiento que tienen durante la práctica deportiva los deportistas que entrena. Consta de catorce ítems que describen situaciones que ocurren durante la práctica deportiva. Un observador externo debe valorar los ítems en una escala de 1 a 5 , donde 5 significa totalmente de acuerdo y 1 totalmente desacuerdo. El instrumento presenta unas adecuadas características psicométricas.

Otros instrumentos para la evaluación de los constructos deportividad y/o no deportividad han sido los registros de observación. El Instrumento de Observación de "Fairplay" en Fútbol (IOFF) (Borrás et al., 2003) o la Plantilla de Observación de Conductas de "Fairplay" (Cruz et al., 1996) pueden ser utilizados, tanto para la evaluación en el deporte profesional como en el deporte en edad escolar. El primero, permite la evaluación de deportistas y árbitro, además de poder ser utilizado a lo largo de un momento temporal. El segundo, permite únicamente la evaluación de los deportistas en un único momento temporal. El instrumento presenta un índice de Kappa de Cohen = .79.

Por otro lado, han sido diversas las investigaciones (González-Oya y Dosil, 2004; Lefebre y Passer, 1974; Olmedilla, Lozano y Andreu, 2001) las que han utilizado el informe, concretamente las actas arbitrales, bien en el deporte profesional, bien en el deporte en edad escolar, para la evaluación de comportamientos inadecuados en deportistas y entrenadores. El juez recoge el número de sanciones que ha mostrado a los agentes señalados mientras realizan la práctica deportiva. Como cada sanción hace referencia a la infracción de algún artículo del reglamento del deporte evaluado, los autores de los estudios previamente mencionados, realizan estadísticas de sanciones para evaluar exclusivamente el tipo de conductas antideportivas que se dan con más frecuencia durante la temporada en deportistas y entrenadores. Los estudios que utilizan las actas arbitrales como instrumento de evaluación no presentan características psicométricas.

\section{Discusión}

El presente artículo muestra la revisión realizada en bases de datos, en publicaciones e informes de federaciones e instituciones deportivas. Concretamente, se realiza un análisis bibliométrico (Spinak, 1996) de los documentos relacionados con instrumentos de evaluación de la deportividad y/o no deportividad en ámbitos deportivos. A partir de diferentes clasificaciones de modalidades deportivas, finalmente se ha optado por una definición de deporte como sistema abierto (Dietrich y Heinemann, 1989; Heinemann, 1991). Los resultados obtenidos en el análisis bibliométrico confirman 
la clasificación de modalidades deportivas propuesta por Willimczik (2009). Por tanto, los instrumentos de evaluación que se han utilizado para evaluar los constructos deportividad, agresividad y violencia, realizan una diferenciación en relación al ámbito que permiten evaluar; deporte profesional, deporte educativo en edad escolar o de ambas modalidades.

A partir del análisis bibliométrico realizado, son escasos los documentos encontrados, los que de forma específica permiten la evaluación de la deportividad, agresividad y violencia en ámbitos deportivos de formación (Bolter y Weiss, 2012; Gibbons, Ebbeck y Weiss, 1995; Gimeno et al., 2007; Greenwell et al., 2001; Horroks, 1979; Lee, 1996; McMahan, 1978). Mayoritariamente, se han elaborado instrumentos que se utilizan para la evaluación de cualquier ámbito deportivo, profesional o en edad escolar. Entre los instrumentos para la evaluación de los constructos deportividad y/o no deportividad, el más utilizado ha sido el cuestionario (Cruz et al., 1996; García Calvo, 2006; Gibbons et al., 1995; Gimeno et al., 2007; Greenwell et al., 2001; Horroks, 1979; Kavussanu y Boardley, 2009; Lakie, 1964; Lee, 1999; McMahan, 1978; Pelegrín, 2005; Vallerand, 1997). En menor medida se utiliza el acta arbitral (González-Oya y Dosil, 2004; Lefebre y Passer, 1974), el registro de observación (Borrás et al., 2003; Cruz et al., 1996; Gibbons et al., 1995) o la entrevista (Gibbons et al., 1995). Por otro lado, los resultados muestran que hay instrumentos que evalúan los constructos objeto de estudio en base a una conceptualización de un listado de: actitudes (Blair, 1985; Case, Greer y Lacourse, 1987; Kavussanu y Roberts, 2001), valores (Lee y Cook, 1990; Lee, 1993; Lee, O'Donoghue y Hodgson, 1990; Torregrosa y Lee, 2000), conductas (García Calvo, 2006; Gimeno, Sáenz, Ariño y Aznar, 2007; Kavussanu y Boardley, 2009). Estas variables psicológicas han sido recogidas en los instrumentos de evaluación elaborados al efecto, de forma separada o de forma conjunta. Es decir, se han derivado bien hacia el constructo deportividad, bien hacia el constructo violencia y agresividad, bien hacia los tres constructos.

\section{Conclusiones}

Entre las características comunes de los 21 instrumentos de evaluación presentados en este artículo, caben destacar: (1) mayoritariamente se utiliza el cuestionario (76.19\%) (2) las herramientas evaluadoras son cumplimentadas por un único agente, el deportista (71.43\%), lo que aumenta el sesgo de las tendencias individuales. Se ignoran variables tales como las influencias sociales, las situaciones antecedentes, el estado personal, otras explicaciones teóricas diferentes al marco conceptual en el que se sustenta el registro de evaluación; (3) tienden a elaborarse para la evaluación de cualquier práctica deportiva (85.71\%); (4) generalmente se utilizan en momentos puntuales $(85.71 \%)$ y no a lo largo de un período temporal (14.29\%); (5) los instrumentos evalúan bien el constructo deportividad (14.28\%), bien el constructo no deportividad (28.57\%), bien ambos constructos de manera conjunta (57.14\%); (6) tienden a poder ser utilizados en cualquier ámbito del deporte (66.66\%). En menor medida se han elaborado herramientas que permitan de manera específica la evaluación de contextos deportivos en edad escolar (33.33\%); (7) algunos instrumentos aportan escasa información en relación al constructo que pretenden medir; (8) la medición de otras variables, además de la deportividad y/o no deportividad, conlleva que dicha medida tenga dificultades a la hora de analizarse psicométricamente.

Entre las características específicas de los 7 instrumentos que de forma específica permiten evaluar los contextos deportivos en edad escolar, caben destacar: (1) el cuestionario es el método de evaluación utilizado (100\%) (Bolter y Weiss, 2012; Gibbons, Ebbeck y Weiss, 1995; Gimeno et al., 2007; Greenwell et al., 2001; Horroks, 1979; Lee, 1996; McMahan, 1978); (2) de forma simultánea permiten la evaluación del constructo deportividad y no deportividad (85.71\%) (Bolter y Weiss, 2012; Gibbons, Ebbeck y Weiss, 1995; Gimeno et al., 2007; Greenwell et al., 2001; Horroks, 1979; McMahan, 1978); (3) se centran en la evaluación de deportistas (42.86\%) (Gibbons, Ebbeck y Weiss, 1995; Horroks, 1979; Lee, 1996) y entrenadores (42.86\%) (Bolter y Weiss, 2012; Greenwell et al., 2001; McMahan, 1978). Son escasos los que permiten evaluar de forma conjunta a entrenadores, deportistas, padres y árbitros (14.28\%) (Gimeno et al., 2007); (4) tienden a evaluar cualquier práctica deportiva (85.71\%) (Bolter y Weiss, 2012; Gibbons, Ebbeck y Weiss, 1995; Greenwell et al., 2001; Horroks, 1979; Lee, 1996; McMahan, 1978). Son escasos los que se centran en evaluar un único deporte (14.28\%) (Gimeno, 2007; Sáenz, 2010); (5) se utilizan en un único momento temporal (85.71\%) (Bolter y Weiss, 2012; Gibbons, Ebbeck y Weiss, 1995; Greenwell et al., 2001; Horroks, 1979; Lee, 1996; McMahan, 1978). Son escasos los instrumentos los que permiten la evaluación a lo largo de un período temporal (14.28\%) (Gimeno et al., 2007; Sáenz, 2010); (6) presentan adecuadas características psicométricas (Bolter y Weiss, 2012; Gibbons, Ebbeck y Weiss, 1995; Gimeno et al., 2007; Greenwell et al., 2001; Horroks, 1979; Lee, 1996; McMahan, 1978).

\section{Aplicaciones prácticas}

Atendiendo a los resultados obtenidos, es deseable que se elaboren instrumentos que permitan evaluar la deportividad y no deportividad de forma conjunta a lo largo de un período temporal, de forma específica en contextos deportivos en edad escolar. Además, específicos para cada deporte, ya que cada uno posee su propia idiosincrasia. La evaluación debe permitir detectar el tipo de actitudes y conductas prosociales 
y antisociales, además de permitir identificar a los agentes que las desarrollan. Finalmente, sería adecuado que los instrumentos que se propongan utilicen escalas multi-ítem, con el propósito de prever posibles respuestas. En definitiva, a partir de una adecuada evaluación de los contextos deportivos en edad escolar, desde un marco ecológico, se hace necesario implementar programas multicomponentes (Gimeno et al., 2011) que dispongan de una metodología precisa, con estrategias y recursos adaptados a la totalidad de agentes que afectan a la realidad de los deportistas. Además, estos programas deben tener en cuenta diferentes situaciones y circunstancias que puedan acontecer en contextos deportivos de formación. Estos programas deberían incluir; 1) acciones de carácter divulgativo y formativo (prevención primaria); 2) acciones de prevención e intervención (prevención secundaria); y 3) acciones de intervención en crisis (prevención terciaria).

\section{Referencias bibliográficas}

1. Bellack, A., Kliebard, H., Hyman, R. y Smith, F. (1966). The language of the classroom. New York: Teachers College, Columbia University Press.

2. Berengüí, R. y Garcés de Los Fayos, E. (2007). Valores en el deporte escolar: estudio con profesores de Educación Física. Cuadernos de Psicología del Deporte, 7(2), 103.

3. Blair, S. (1985). Professionalisation of attitude towards play in children and adults. Research Quarterly, 56(1), 82-83.

4. Blázquez, D. (1999). La iniciación deportiva y el deporte escolar. Barcelona: INDE.

5. Bolter, N. y Weiss, M. (2012). Coaching for Character: Development of the Sportsmanship Coaching Behaviors Scale (SCBS). Sport, Exercise, and Performance Psychology, 1(2), 73-90.

6. Borrás, A., Palou, P., Ponseti, F. y Cruz, J. (2003). Promoción de la deportividad en el fútbol en edad escolar: Evaluación de los comportamientos relacionados con el fair-play. En S. Márquez (Ed.), Psicología de la actividad física y el deporte: perspectiva latina (pp. 207-217). Universidad de León: Secretariado de Publicaciones y Medios Audiovisuales.

7. Bredemeier, B. y Shields, D. (1984). The utility of moral stage analysis in the investigation of athletic aggression. Sociology of Sport Journal, 1, 138-149.

8. Brohm, J. (1976). Sociología politica del deporte. México: Fondo de Cultura Económico.

9. Cagigal, J.M. (1983). El cuerpo y el deporte en la sociedad moderna. Papers Revista de Sociología, 22, 145- 156.

10. Cagigal, J.M. (1981): Oh deporte. Anatomía de un gigante. Miñón. Valladolid.

11. Case, B., Greer, H. y Lacourse, M. (1987). Moral judgment development and perceived legitimacy of spectator behavior in sport. Journal of Sport Behavior, 10, 147-156.

12. Conroy, D. y Coatsworth, J. (2006). Coaching training as a strategy for promoting youth social development. The Sport Psychologist, 20, 128-144.

13. Cruz, J., Capdevilla, L., Boixadós, M., Pintanel, M., Alonso, C., Mimbrero, J. y Torregrosa, M. (1996). Identificación de conductas, actitudes y valores relacionadas con el fair play en deportistas jóvenes. Investigaciones en ciencias del deporte, 9, 37-87.

14. Dietrich, K. y Heinemann, K. (1989). Der Nicht-sporliche Sport. Schondorf: Hofmann Verlag.

15. Dosil, J. (2003). La función educativa del árbitro y juez deportivo. En F. Guillén (Dir.). Psicología del arbitraje y el juicio deportivo (pp. 133160). Barcelona: INDE.

16. Dunn, J. y Dunn, J. (1999). Goal orientations, perceptions of aggression, and sportspersonship in elite male youth ice hockey players. The Sport Psychologist, 13, 183-200.

17. Durán, J. (1996). Deporte, violencia y educación. Revista de Psicología del Deporte, 9(10), 103-109.
18. García, T. (2006). Motivación y comportamientos adaptativos en jóvenes deportistas. Tesis doctoral no publicada. Cáceres: Universidad de Extremadura.

19. Garmezy, N. (1983). Stressors of childhood. En N. Garmezy y M. Rutter (Eds.), Stress, coping and development in children (pp.43-84). Nueva York: McGraw-Hill.

20. Gibbons, S., Ebbeck, V. y Weiss, M. (1995). Fair play for kinds: effects on the moral functioning of children in psysical education. Research Quarterly for Exercise and Sport, 66, 247-255.

21. Gill, D. (2000). Psychological dynamics of sport and exercise. Campaign, IL: Human Kinetics.

22. García Ferrando, M. (2001). Los españoles y el deporte: prácticas y comportamientos en la última década del siglo XX. Madrid: CSD y Ministerio de Educación, Cultura y Deporte.

23. Gimeno, F., Sáenz, A., Ariño, J. y Aznar, M. (2007). Deportividad y violencia en el fútbol base: un programa de evaluación y de prevención de partidos de riesgo. Revista Psicología del Deporte 16(1), 103-118.

24. Gimeno, F., Sáenz, A., Gutiérrez, H., Lacambra, D., París, F. y Ortiz, P. (2011). El programa multicomponente "Juguemos limpio en el deporte base: Desarrollo y aportaciones en la década de 2000-2010". II Seminario Nacional Fomento de la Deportividad y Prevención de la Violencia en el Deporte en Edad Escolar. Vitoria-Gasteiz, España, 24-26 de Marzo.

25. Goldstein, A. (1999). Aggression reduction strategies: effective and ineffective. School Psychology Quarterly, 14(1), 40-58.

26. González-Oya, J. y Dosil, J. (2007). La Psicología del árbitro de fútbol. A Coruńa: Toxosoutos.

27. González-Oya, J. y Dosil, J. (2004). Características psicológicas de árbitros de fútbol. Cuadernos de Psicología del Deporte, 4, 53-66.

28. Greedorfer, S. (1992). Sport socialization. En T. Horn (Ed.), Advances in short psychology (pp. 201-218). Champaign, IL: Human Kinetics.

29. Greenwell, T., Geist, A., Mahony, D. Jordan, J. y Pastore, D. (2001). Characteristics of NCAA conference codes of ethics. International Journal of Sport Management, 2, 108-124.

30. Harwood, C. G., Cumming, J. y Fletcher, D. (2004). Motivational profiles and psychological skills use within elite youth sport. Journal of Applied Sport Psychology, 16, 318-332.

31. Heinemann, K. (1991). Tendencias de la investigación social aplicada al deporte. En Acta del Congreso politicas deportivas e investigación social. Pamplona: Gobierno de Navarra, Departamento de Educación Cultura y Deporte.

32. Heinemann, K. (1986). The future of sports. Challenge for the science of sport. International Review for the Sociology of Sport, 4(21), 271-285.

33. Horn, T. (2008). Coaching effectiveness in the sport domain. En T. Horn (Ed.), Advances in sport psychology (pp. 239-267). Champaign, IL: Human Kinetics.

34. Horrocks, R. (1979). The relationship of selected prosocial play behaviors in children to moral reasoning, youth sports participation, and 
perception of sportsmanship. Tesis Doctoral. University of North Carolina at Greensboro.

35. Kavussanu, M. y Boardley, I. (2009). The prosocial and antisocial behavior in sport scale. Journal of Sport \& Exercise Psychology, 31, 97-117.

36. Kavussanu, M. y Roberts, G. (2001). Moral functioning in sport: an achievement goal perspective. Journal of sport and Exercise Psychology, 23, 37-54.

37. Lakie, W. (1964). Expressed attitudes of various groups of athletes toward athletic competition. Research Quarterly, 35, 497-503.

38. Lee, M. (1996). Young people, Sport and ethics: an examination of fairplay in youth sport. Technical Report to the Research Unit of the Sports Council: Londres.

39. Lee, M. (1993). Coaching children in sport. Londres: Spon.

40. Lee, M. y Cook, C. (1990). Review of the literature on fairplay with special reference to children's sport. Strasbourg: Council of Europe.

41. Lee, M., O’Donohue, R. y Hodson, D. (1990). Ethical issues in sport. The measurement of values in sport: a review of methods for a proposed trans-european Project. Strasbourg: Consejo de Europa.

42. Lefebre, L. y Passer, M. (1974). The effects of game location and importante on aggression in team sport. International Journal of Sport Psychology, 5(2), 102-110.

43. Leo, F., Sánchez, P., Sánchez, D., Amado, D. y García, T. (2009). Interacción entre la percepción del comportamiento de los padres y los comportamientos deportivos en edades de iniciación. Acción Psicológi$c a, 6(2), 55-62$.

44. Liberati, A., Altman, D.G., Tetzlaff, J., Mulrow, C., Gotzsche, P.C., Loannidis, J.P., Clarke, M., Devereaux, P.J., Kleijnen, J. y Moher, D. (2009). The PRISMA statement for reporting systematic reviews and meta-analyses of studies that evaluate healthcare interventions: explanation and elaboration. British Medical Journal, 339, 2700.

45. Martens, R. (1982). Kids sports: A den of iniquity or land of promise. Champaign, IL: Human Kinetics.

46. Martín-Albo, J., Núñez, J., Navarro, J. y González, V. M. (2006). Validación de la versión española de la escala multidimensional de orientaciones a la deportividad. Revista de Psicología del Deporte, 15(1), 9-22.

47. Matveev, L. (1980). Fundamentos del Entrenamiento Deportivo. Moscú: MIR.

48. McMahan, R. (1978). The development of an instrument for assessing sportsmanship attitudes. Unpublished doctoral dissertation. University of Tennessee, Knoxville.

49. McPherson, B. y Brown, B. (1988). The structure, processes, and consequences of sport for children. En F. Smoll, R. Magill y M. Ash (Eds.), Children in sport (pp. 265-286). Champaign, IL: Human Kinetics.

50. Miller, B., Roberts, G. y Ommundsen, Y. (2005). Effect of perceived motivational climate on moral functioning, team moral atmosphere perceptions, and the legitimacy of intentionally injurius acts among competitive youth football players. Psychology of Sport and Exercise, 6(4), 461-477.

51. Moreno, J. A. (2008). Importancia de la práctica físico-deportiva y del género en el autoconcepto físico de los 9 a los 23 años. International Journal of Clinical and Health Psychology, 8(1), 171-183.

52. Nicholls, J. G. (1989). The competitive ethos and democratic education. Cambridge, MA: Harvard University Press.

53. Olmedilla, A., Lozano, F. y Andreu, M. (2001). Intervención psicológica para la eliminación o disminución de sanciones: una experiencia en fútbol semiprofesional. Revista de Psicología del Deporte, 10(2), 157175.

54. Pelegrín, A. y Garcés de Los Fayos, E. (2007). Agresión y violencia en el deporte. Sevilla: Wanceulen.

55. Pelegrín, A. (2005). Detección y valoración de la incidencia de las actitudes antideportivas durante la competición. Cuadernos de Psicología del Deporte, 5(1-2), 133-142.

56. Ponseti, F.J., Palou, P., Borrás, P.A., Vidal, J., Cantallops, J., Ortega,
F., Boixadós, M., Sousa, C., García-Calvo, T., García-Mas, A. (2012). El cuestionario de disposición al engaño en el deporte (CDED): su aplicación a jóvenes deportistas. Revista de Psicología del Deporte, 21(1), 75-80.

57. Puig, N. y Heinemann, K. (1991). El deporte en la perspectiva del año 2000. Papers. Revista Sociología, 38, 123-141.

58. Ramírez, A., Alonso-Arbiol, I., Falcó, F. y López, M. (2006). Programa de intervención psicológica con árbitros de fútbol. Revista de Psicología del Deporte, 15, 311-325.

59. Rato, M., Ley, C., y Durán, J. (2009). Derechos humanos y cooperación para el desarrollo en y a través del deporte. En J. Moreno y D. González-Cutre (Eds.), Deporte, Intervención y Transformación Social (pp. 13-58). Río de Janeiro: Fondo de Cultura económica.

60. Roberts, G. (1995). Motivation in sport and exercise: conceptual constraints and convergence. En G. C. Roberts (Ed.), Motivation in sport and exercise (pp. 3-30). Campaign, IL: Human Kinetics.

61. Robles, J., Abad, M. T. y Giménez, F. J. (2009). Concepto, características y clasificaciones del deporte actual. EFDeportes Revista Digital, 14, 138.

62. Romand, P., Pantaléon, N. y Cabagno, G. (2009). Age Differences in Individuals. Cognitive and Behavioral Moral Functioning Responses in Male Soccer Teams. Journal of Applied Sport Psychology, 21(1), 49-63.

63. Rutter, M. (1990). Psychosocial resilience and protective mechanisms. En J. Rolf, A. Masten, D. Cicchetti, K. Nuechterlein y S. Weintraub (Eds.), Risk and protective factors in the development of psychopa theology (pp. 118-214). Nueva York: Cambridge University Press.

64. Sáenz, A. (2010). Deportividad y violencia en el fútbol base. Tesis doctoral no publicada. Zaragoza: Universidad de Zaragoza.

65. Slutzky, C. B. y Simpkins, S. D. (2009). The link between children's sport participation and selfesteem: exploring the mediating role of sport self-concept. Psychology of Sport and Exercise, 10(3), 381-389.

66. Spinak, E. (1996). Diccionario enciclopédico de bibliometría, cienciometría e infometría. Venezuela: UNESCO.

67. Stephens, D. y Bredemeier, B. (1996). Moral atmosphere and judgments about aggression in girl's soccer: relationships among moral and motivational variables. Journal of Sport and Exercise Psychology, 18, 158-173.

68. Stewart, M. y Corbin, C. (1989). Self-confidence of young girls in psysical activity and sport. Runner, 6, 38-41.

69. Schwartz, S. (1996). Value priorities and behaviour: applying a theory of integrated value systems. En C. Seligman, J. Olson y M. Zanna (Eds.), The Psychology of Values: The Ontario Symposium (Vol. 8, pp. 1-24). Mahwah, NJ: Lawrence Erlbaum.

70. Simon, R. (1991). Fair Play. Sports, Values Society. Estats Units: Westwiew

71. Teques, P. y Serpa, S. (2009). Implicación parental: adaptación de un modelo teórico al deporte. Revista de Psicología del Deporte, 18(2), 235252.

72. Tenenbaum, G., Stewart, E., Singer, R y Duda, J. (1997). Aggression and Violence in Sport: An ISSP Position Stand. The Sport Psychologist, 11, 1-7.

73. Tenenbaum, G., Fogarty, G. y Jackson S. (1999). The "flow" experience: a Rasch analysis of Jackson's "Flow" State Scale. Journal of Outcome Measurement, 3(3), 278-94.

74. Torregrosa, M. (2002). Estudio de valores, motivaciones y emociones de los aficionados al fútbol: una aproximación a partir de la integración de metodologías. Bellaterra: UAB.

75. Torregrosa, M. y Lee, M. (2000). El estudio de los valores en psicología del deporte. Revista de Psicología del Deporte, 9(1-2), 71-83.

76. Turnbull, B. (2002). Program theory building: A strategy for deriving cumulative evaluation knowledge. American Journal of Evaluation, 23 , 275-90.

77. Ullrich-French, S. y Smith, A. (2009). Social and motivational pre- 
dictors of continued youth sport participation. Psychology of Sport and Exercise, 10, 87-95.

78. Urrútia, G., y Bonfill, X. (2010). PRISMA declaration: A proposal to improve the publication of systematic reviews and meta-analyses. $\mathrm{Me}$ dicina Clinica, 135, 507-5011.

79. Vallerand, R., Deshaies, P. y Cuerrier, J. (1997). On the Effects of social Context on Behavioral Intentions of Sporsmanship. Sport Psycho$\log y, 28,126-140$.

80. Vallerand, R. (1997). Toward a Hierarchical Model of Intrinsic and Extrinsic Motivation. En M. Zanna (Ed.), Advances in experimental social psychology (pp. 271-360). NewYork: Academic Press.

81. Vallerand, R. y Bissonnette, R. (1992). Intrinsic, extrinsic and amotivational styles as predictors of behaviour; a prospective study. Journal of Personality, 60, 599-620.

82. Vargas, R. (2007). Diccionario de Teoría del Entrenamiento Deportivo. México D.F.: Universidad Nacional Autónoma de México.

83. Volkamer, M. (1971). Zur Aggresivität in konkurrenz-orientierten sozialen Systemen. Eine Untersuchung an Fussballpunktspielen (Agre- sión en sistemas sociales orientados a la rivalidad. Una investigación sobre el fútbol). Sportwissenschaft,1, 33-64.

84. Weinberg, R. y Gould, D. (2010). Foundations of Sport and Exercise Psychology. Canada: Human Kinetics.

85. Weiss, M. y Bredemeier, B. (1991). Moral development in sport. En K. Pandolf y J. Holloszy (eds.), Exercise and Sport Science Reviews (pp. 331-378). Champaign, IL: Human Kinetics.

86. Willimczik, K. (2009). El Deporte en Alemania: de una categoría ontológica a un concepto del presente. Apunts Educación Física y Deporte, 97, 48-60.

87. Wuerth, S., Lee, M. \& Alfermann, D. (2004). Parental Involvement and athletes career in youth Sport. Psychology of Sport and Exercise, 5, 21-33.

88. Zinsser, N., Bunker, L. y Williams, J. (2001). Cognite techniques for building confidence and enhancing performance. En J.M. Williams (ed.), Applied sport psychology: personal growth to Peak performance, Mountain View, CA: Mayfield. 